\title{
Capturing multi-regime combustion in turbulent flames with a virtual chemistry approach
}

\author{
Giampaolo Maio ${ }^{\mathrm{a}}$, Mélody Cailler ${ }^{\mathrm{a}, \mathrm{b}}$, Nasser Darabiha ${ }^{\mathrm{a}}$, Benoît Fiorina ${ }^{\mathrm{a}, *}$ \\ ${ }^{a}$ Laboratoire EM2C, CNRS, CentraleSupélec, Université Paris-Saclay, 3 rue Joliot Curie \\ 91192 Gif Sur Yvette cedex, France \\ ${ }^{b}$ Safran Tech, Modelling $\&$ Simulation, Rue des Jeunes Bois, Châteaufort, 78114 \\ Magny-Les-Hameaux, France
}

\section{Abstract}

Multiple flame regimes are encountered in industrial combustion chambers, where premixed, stratified and non-premixed flame regions may coexist. To obtain a predictive tool for pollutant formation predictions, chemical flame modeling must take into account the influence of such complex flame structure. The objective of this article is to apply and compare two reduced chemistry models on both laminar and turbulent multi-regime flame configurations in order to analyze their capabilities in predicting flame structure and $\mathrm{CO}$ formation. The challenged approaches are i) a premixed flamelet-based tabulated chemistry method, whose thermochemical variables are parametrized by a mixture fraction and a progress variable, and ii) a virtual chemical scheme which has been optimized to retrieve the properties of canonical premixed and non-premixed 1-D laminar flames. The methods are first applied to compute a series of laminar partially-premixed methane-air counterflow flames. Results are compared to detailed chemistry simulations. Both ap-

\footnotetext{
${ }^{*}$ Corresponding author: Benoît Fiorina

Email address: benoit.fiorina@centralesupelec.fr (Benoît Fiorina)
} 
proaches reproduced the thermal flame structure but only the virtual chemistry captures the $\mathrm{CO}$ formation in all ranges of equivalence ratio from stoichiometry premixed flame to pure non-premixed flame. Finally, the two chemical models combined with the Thickened Flame model for LES are challenged on a piloted turbulent jet flame with inhomogeneous inlet, the Sydney inhomogeneous burner. Mean and RMS of temperature and CO mass fraction radial profiles are compared to available experimental data. Scatter data in mixture fraction space and Wasserstein metric of numerical and experimental data are also studied. The analyses confirm again that the virtual chemistry approach is able to account for the impact of multi-regime turbulent combustion on the $\mathrm{CO}$ formation.

Keywords:

Reduced Chemistry, Turbulent combustion modeling, Pollutant formation, Large-Eddy Simulation

\section{Colloquium: TURBULENT FLAMES}

Total length of the paper: 7.5 pages

The authors will pay color reproduction charges if applicable.

\section{Introduction}

Many practical combustion chambers promote the development of multiplemode turbulent combustion regimes where premixed, non-premixed and partiallypremixed flame structures coexist [1]. In such environment, the description of detailed chemical phenomena, at a CPU cost compatible with industrial constraints, is a critical modeling challenge [2]. 
By taking benefit of the increasing computational resources, reduced mechanisms are now employed to conduct simulations of practical combustion chambers $[3,4]$. They allow to describe complex combustion phenomena sensitive to detailed chemistry. However, because of the high number of species and the numerical stiffness of the system, the systematic use of reduced mechanisms in a daily design process of combustion chamber remains expensive.

To save computational time, tabulated chemistry suggests to build-up, prior to CFD computations, chemical look-up tables where all thermo-chemical information are stored. The tabulation strategy is very efficient for conventional combustion systems characterized by one main flame regime [5-8] but its extension towards multi-regime combustion requires more coordinates than the conventional ones that increases the complexity of the methodology [9-11]. Virtual chemistry has recently been developed as an alternative to tabulated chemistry [12-14]. The originality of the method relies on building-up a reduced chemical scheme using virtual species and reactions whose thermo-chemical properties are calibrated to capture quantities of interest. As for tabulated chemistry, the range of validity of the virtual chemistry method depends on the set of target flames retained to optimize the chemical parameters. Recent developments showed, for instance, that CO formation is well predicted in both premixed and diffusion laminar flames if premixed and non-premixed elements are targeted during the optimization step [13]. The ability of the method to capture the chemical structure of multi-regime turbulent flame remains however unexplored.

An interesting configuration to challenge the ability of turbulent com- 
bustion model to capture multi-regime flame regime is the Sydney Inhomogeneous burner [15-17]. Selected as a target flame for the TNF workshop [18], this configuration has recently been studied by many groups using various chemistry and turbulent combustion modeling strategies [16, 19-26]. Most studies combined tabulated chemistry with presumed [19-22, 24] or transported [26] Probability Density Functions. A few studies also employed transported complex chemistry combined with transported PDF [20, 25]. Recently, a hybrid model, combining reduced transported chemistry and tabulated chemistry with PDF transport, has been introduced [26] to limit the computational cost. A comparison between several modeling approaches, performed during the TNF workshop [18], shows that it is difficult to capture CO production because it is highly sensitive to the flamelet ingredients used for chemistry reduction or tabulation.

The objective of the present work is to compare virtual chemistry against premixed-based tabulated chemistry in a multi-regime turbulent combustion regime. The combustion modeling strategies are presented in Sec. 2 and challenged on laminar multi-regime counterflow flames in Sec. 3. Details on the experimental and numerical set-ups of the jet flame with homogeneous inlets are provided in Sec. 4. Numerical results of the turbulent flame are compared against measurements n Sec. 5, giving a special emphasis on radial statistics, scatter plots and Wasserstein metric [21]. 


\section{Chemistry modeling}

\subsection{FPI tabulated chemistry}

The FPI (Flame Prolongation of ILDM) [7] combustion chemistry model approximates the chemical space covered by turbulent flames by a reduced manifold composed by 1-D laminar premixed flamelets. Flame thermochemical quantities $\psi$ are then tabulated as a function of a reduced set of variables which, under adiabatic conditions, are the mixture fraction $Z$ and the

progress variable $Y_{c}$, according to the relation $\psi=\psi^{*}\left[Y_{c}, Z\right]$. The progress variable, defined as $Y_{c}=Y_{\mathrm{CO}_{2}}+Y_{C O}$, tracks the transition from fresh to burnt gases. The mixture fraction dimension $Z$ is covered by varying the fresh gas equivalence ratio of the premixed flames within the range of flammability. As discussed in [27], this approach is adapted to partially-premixed flames as soon as the level of stratification remains moderate. However, when multi-regime flame structures are encountered, FPI fails to reproduce diffusion flame regimes and causes departures in regard with detailed chemistry, which are especially visible on CO mass fraction [11].

\subsection{Virtual chemistry}

Virtual chemistry has recently been introduced $[12,13]$ as an alternative to flamelet-based tabulated chemistry. The concept relies on the design of chemical mechanisms made of virtual species and reaction whose thermo-chemical properties are calibrated. Instead of being tabulated, flame archetypes constitute a learning database retained to train optimized virtual mechanisms. Different ingredients, such as premixed and non-premixed 
flamelets may be included in the learning database so that the virtual chemical scheme is able to track various combustion regimes.

A virtual chemical scheme is made of (i) a main reduced mechanism, dedicated to temperature and heat release prediction, and (ii) a series of standalone satellite reduced sub-mechanisms, each being dedicated to a specific pollutant species prediction [13]. It has been shown in [12] that the following 2-step main virtual mechanism accurately captures the heat release and the flame temperature for both premixed and non-premixed combustion regimes:

$$
\begin{aligned}
\alpha_{F}^{v} F+\alpha_{O x}^{v} O x & \rightarrow \alpha_{I}^{v} I & \left(R_{1}^{T}\right) \\
\alpha_{I}^{v} I & \rightarrow \sum_{k=1}^{N_{P}^{v}} \alpha_{P_{k}}^{v} P_{k} & \left(R_{2}^{T}\right)
\end{aligned}
$$

where $F$ and $O x$ denote the fuel and oxidizer species respectively. Burnt gases mixture is composed of $\mathrm{N}_{P}$ virtual products $P_{k}$ whose thermochemical properties are optimized to recover thermodynamic equilibrium. CO production is modeled by the following 3-step sub-mechanism including 3 additional species:

$$
\begin{aligned}
\alpha_{F}^{v} F+\alpha_{O x}^{v} O x & \rightarrow \alpha_{C O}^{v} C O+\left(1-\alpha_{C O}^{v}\right) V_{1} & & \left(R_{1}^{C O}\right) \\
F+V_{1} & \rightarrow F+C O & & \left(R_{2}^{C O}\right) \\
C O & \leftrightarrow V_{2} & & \left(R_{3}^{C O}\right)
\end{aligned}
$$

The optimization procedure of both main and $\mathrm{CO}$ virtual mechanisms, detailed in [13], is applied to ensure an accurate prediction of flame structure in multi-regime combustion. 


\subsection{Comments on multi-regime combustion}

Tabulated chemistry could be suitable for multi-regime flame when both premixed and non-premixed flamelet ingredients are combined in the same look-up table. However solutions have to solve the projection of species balance equations either into a restricted subset of the composition space $[9,10]$ or to use 1-D partially-premixed flamelets to generate a chemical look-up table [11]. Therefore supplementary control variables should be introduced to generate the chemical table. But such addition increases the complexity of the methodology and raises questions regarding its relevance for the simulations of industrial devices. Unlike to tabulated chemistry, virtual chemistry does not need to identify the most appropriated coordinates on which evolves the reduced chemical subspace. Therefore, the difficult projection of different flame archetypes in a reduced manifold is bypassed by the optimization process of the virtual chemical mechanisms.

\section{Partially-premixed laminar flame simulations}

FPI and virtual chemistry are first challenged on laminar counterflow flame configurations. A methane/air mixture characterized by an equivalence ratio $\phi_{F}($ at $x=+\infty)$ is opposed to an air stream (at $\left.x=-\infty\right)$. By

varying the fuel stream equivalence ratio, a whole range of non-premixed and partially-premixed flame is covered [27]. Reference solutions are obtained with the GRI3.0 [28] detailed mechanism and mixture-averaged transport model. The FPI look-up table is generated from an ensemble of premixed flame solutions whereas both non-premixed and premixed elements are targeted during the virtual mechanism calibration [13]. 
FPI and virtual chemistry solutions, computed using the REGATH solver [29], are compared in Fig. 1 against the detailed chemistry reference solutions for three fuel/air stream equivalence ratio equals to $\phi_{F}=1, \phi_{F}=4$ and $\phi_{F}=$ $+\infty$ (non-premixed flame), respectively. For each configuration, the strain rate is constant and equal to $150 \mathrm{~s}^{-1}$. Temperature profiles predicted by both FPI and virtual chemistry match well the reference solutions for the three configurations. Differences are however noticeable on the CO mass fraction. For $\phi_{F}=1$, FPI and virtual chemistry recover fairly well the CO mass fraction profiles, whose peak amplitude is well captured. A secondary peak is however incorrectly predicted by the FPI solution at the air stream side close to $x=0 \mathrm{~mm}$. A departure between the tabulated chemistry solution and the reference solution, visible at $\phi_{F}=4$, is severely emphasized for the pure diffusion flame $\left(\phi_{F}=+\infty\right)$. Being optimized on both premixed and non-premixed flame archetype, the virtual chemical mechanism reproduces the CO mass fraction for both $\phi_{F}=4$ and $\phi_{F}=+\infty$. After demonstrating the ability of virtual chemistry to capture $\mathrm{CO}$ formation in both partiallypremixed and non-premixed flames, the method is now applied on a turbulent multi-regime flame.

\section{The piloted turbulent jet flame with inhomogeneous inlets con- figuration}

\subsection{Experimental configuration}

The burner assembly [15] is shown in Fig. 2. It consists of two concentric tubes surrounded by a pilot annulus, centered in a wind tunnel supplying a co-flowing air stream at constant velocity of $15 \mathrm{~m} . \mathrm{s}^{-1}$. The fuel and air are fed 

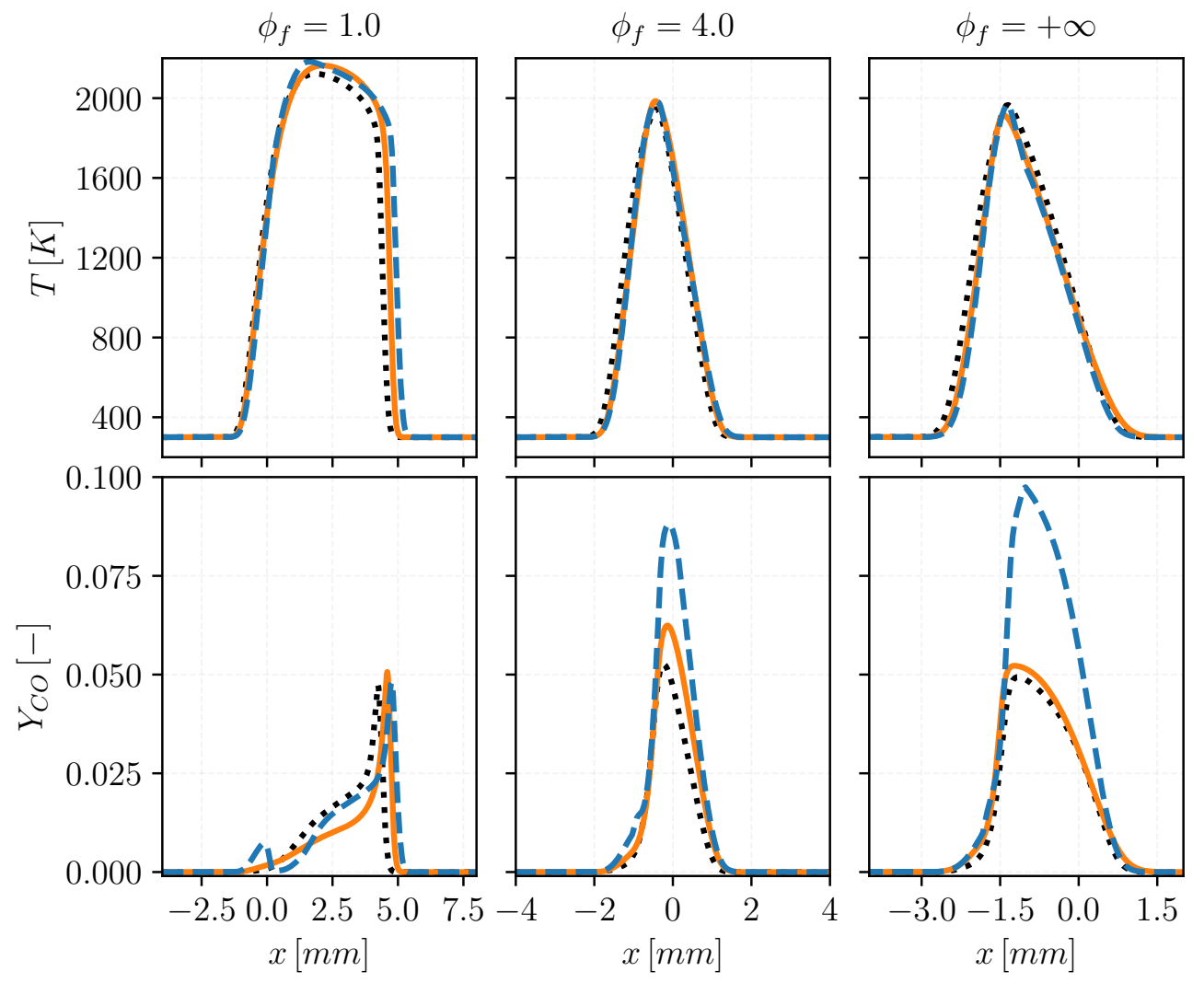

Figure 1: Temperature and CO mass fraction profiles for counterflow laminar flames for different methane/air side equivalence ratios. Dotted black lines: detailed chemistry. Dashed blue lines: FPI-tabulated. Solid orange lines: virtual chemistry. 
from the inner and outer tube, respectively. The outer tube exit of diameter $\mathrm{D}=7.5 \mathrm{~mm}$ constitutes the air/fuel mixture injection inside the combustion chamber. The pilot mixture has been designed to have the same $\mathrm{C} / \mathrm{H}$ ratio and adiabatic flame temperature as a stoichiometric methane/air mixture. The central pipe can be recessed upstream of the burner exit plane varying therefore the quality of mixing between fuel and air before entering the combustion chamber. For a sufficiently large recession distance the mixture is nearly homogeneous at the burner exit. Here, an intermediate recession distance of $75 \mathrm{~mm}$, leading to significant equivalence ratio inhomogeneities at the burner exit, is retained as it promotes multi-regime combustion regime [17]. The main jet bulk velocity is $80 \mathrm{~m} \cdot \mathrm{s}^{-1}$.

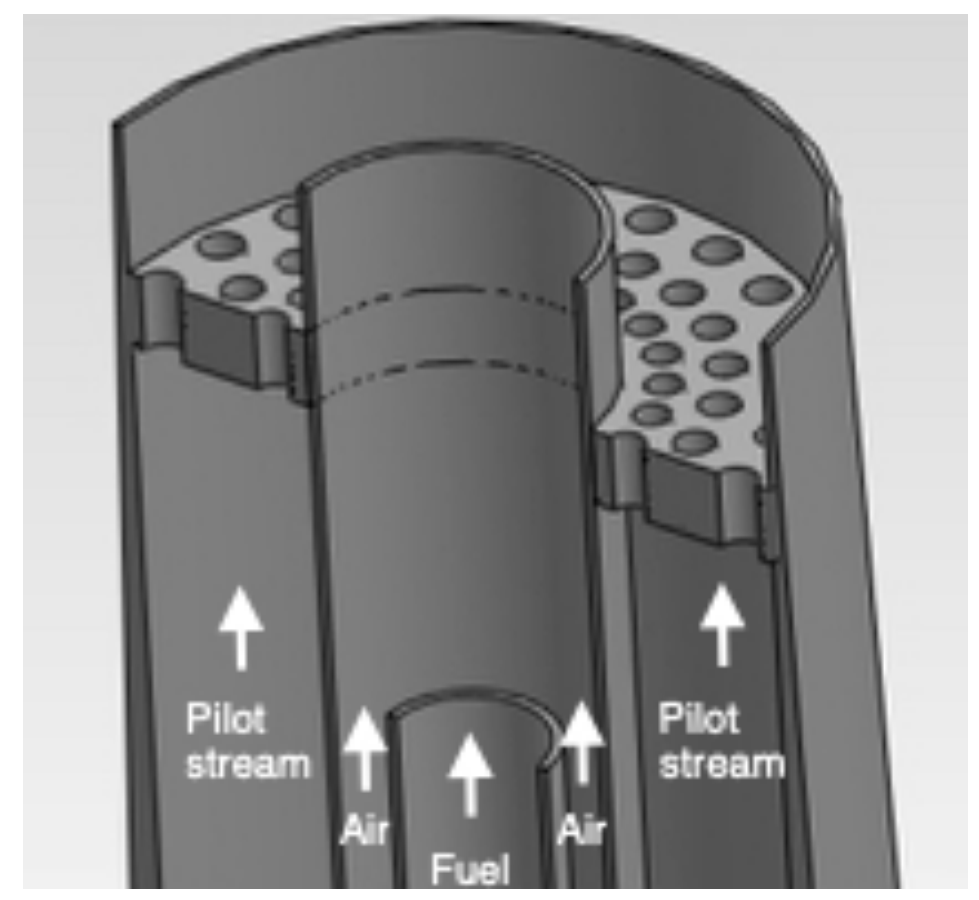

Figure 2: Schematic view of the burner geometry (from [15]) 


\subsection{Numerical set-up}

Non-reactive LES of the mixing process between fuel and air in the mixing tube has been performed by Princeton University [22]. Unsteady solutions of these simulations constitute a temporally and spatially-resolved numerical database that is used to prescribe the main inlet boundary conditions, in terms of flow velocity and mixture fraction.

The computational domain considered for the turbulent flame simulation begins one diameter $(\mathrm{D}=7.5 \mathrm{~mm})$ upstream of the burner exit plane. Calculations are performed with the unstructured finite-volume low Mach number code YALES2 [30]. A centered $4^{\text {th }}$-order scheme in space and time is used for the numerical integration of the convective terms. The sub-grid Reynolds stresses tensor is closed with the WALE model [31]. The tetrahedral mesh features 10.1 M nodes and the mesh size varies from $0.015 \mathrm{~mm}$, in the burner lips region, to $5 \mathrm{~mm}$ far from the flame zone. In the flame region, from the burner exit to $15 \mathrm{D}$ downstream, a characteristic grid size of $0.2 \mathrm{~mm}$ is used.

Both FPI and virtual chemistry combustion chemistry models are coupled with the LES flow governing equations using a dynamic formulation of the Thickened Flame model for LES (TFLES) [32, 33]. Flame sensor definitions are adapted to both tabulated and virtual chemistry models so that the thickening is only applied in the flame reactive layer. Unresolved flame

wrinkling is closed by the Charlette model [34] with a constant $\beta$ parameter equal to 0.5 .

Whereas TFLES and the flame wrinkling model have been designed to capture the propagation speed of turbulent premixed flame $[32,33]$, their suitability to non-premixed flame remains questionable as they may alter 
chemical diffusion flame structure. It is however expected that the bias due to deficiency in turbulent/chemistry interaction modeling is similar in both virtual and tabulated chemistry approaches. The conclusions on the suitability of chemical reduction methods for multi-regime combustion, which is the main objective of this article, will therefore remain valid.

\section{Results}

\subsection{Mixing process and global flame properties}

Figure 3 shows a comparison between numerical and experimental mixture fraction radial profiles extracted at three axial distances from the burner exit, corresponding to $\mathrm{z}=1 \mathrm{D}, 5 \mathrm{D}$ and $15 \mathrm{D}$, respectively. For both chemistry models, numerical mean and RMS mixture fraction profiles compare well to experiments. However, at $\mathrm{z}=5 \mathrm{D}$ and $\mathrm{z}=15 \mathrm{D}$, numerical mean mixture fraction radial profiles are slightly too diffuse while the RMS peak of mixture fraction is slightly underestimated in the shear layer between the main jet and the pilot stream. This discrepancy is attributed to the difficulty to capture the turbulent jet mixing, strongly influenced by shear layer instabilities [35] and by inflow conditions [18].

Barlow et al. [17] revealed in their experiments that pilot gases do not reach equilibrium conditions at the burner exit. The pilot composition and temperature have been adjusted in both FPI and virtual chemistry frameworks to match mean pilot bulk temperature measured at $\mathrm{z}=1 \mathrm{D}$. The radial temperature profiles plotted in Fig. 4 show that both mean and RMS are cor-

rectly reproduced, especially in the virtual chemistry simulation. For both chemical models, discrepancies are however observed further downstream, at 


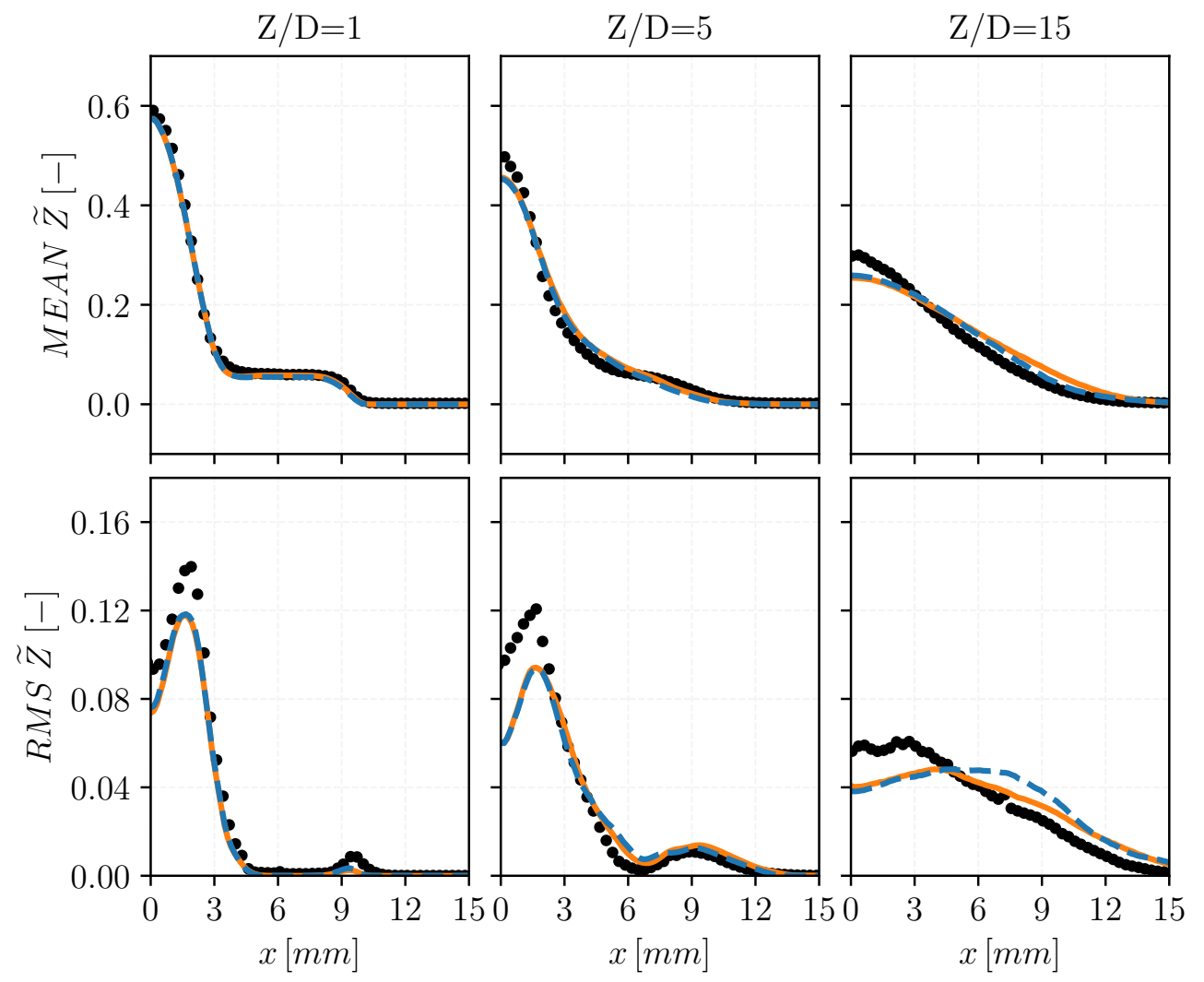

Figure 3: Mean mixture fraction (top) and RMS (bottom) radial profiles. Comparison of the FPI (Dashed lines) and virtual chemistry (Solid lines) results against experimental data (Circle) at three different distances from the injector. 


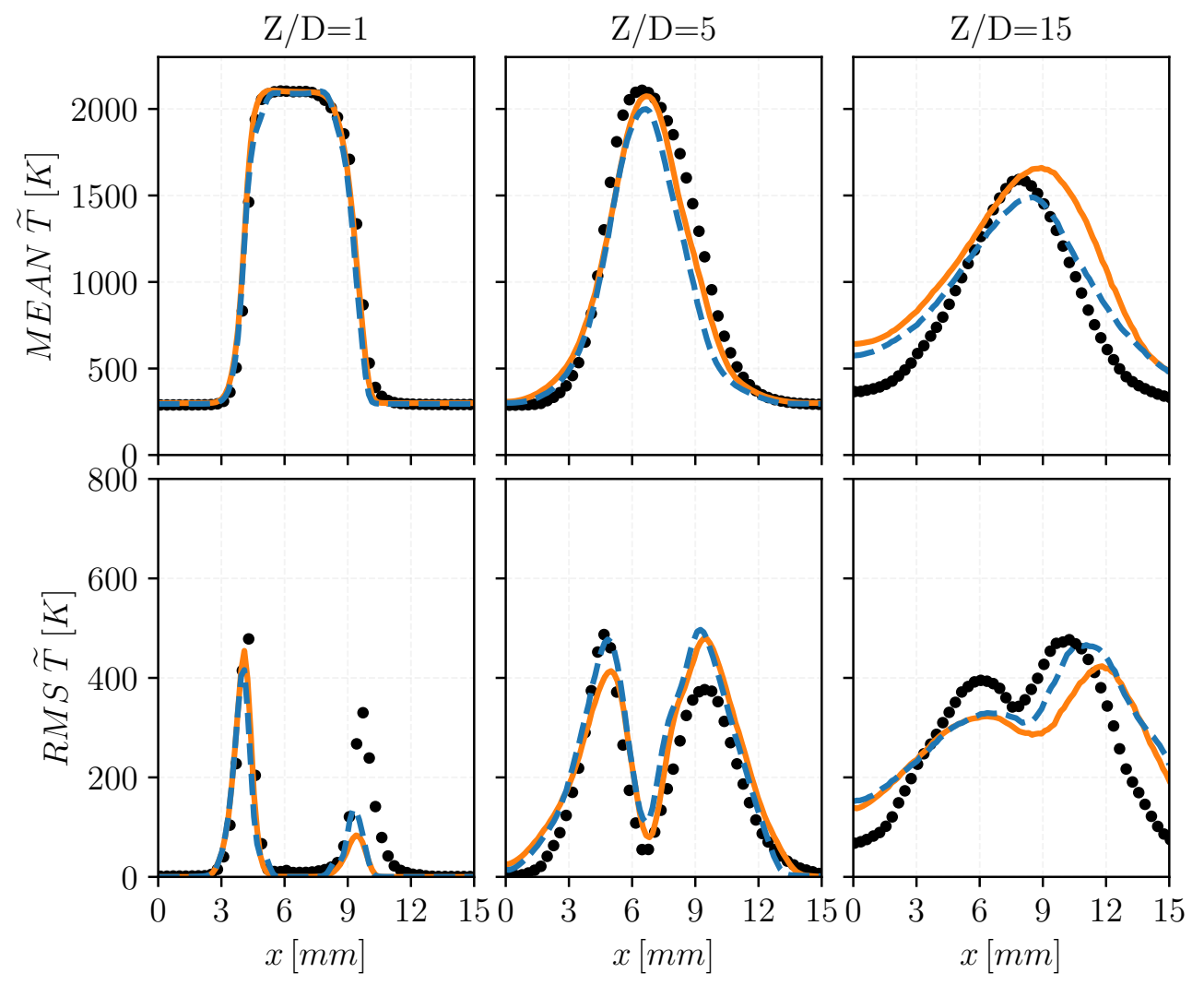

Figure 4: Mean temperature (top) and RMS (bottom) radial profiles. Comparison of the FPI (Dashed lines) and virtual chemistry (Solid lines) results against experimental data (Circle) at different three distances from the injector. 
$\mathrm{z}=15 \mathrm{D}$. This departure is mainly due to the turbulent mixing misprediction previously discussed.

To further analyze the chemical structure of the turbulent flame, scatter plots of temperature versus mixture fraction are plotted in Fig. 5 at horizontal planes located at $\mathrm{z}=1 \mathrm{D}, 5 \mathrm{D}$ and 15D. 150,000 samples are plotted for both simulations and experiments. The vertical dashed dotted line indicates the stoichiometric mixture fraction value $(Z=0.055)$. To highlight the relative importance of highly populated regions, scatter plots are colored by the local density of points. At $\mathrm{z}=1 \mathrm{D}$, the experimental scatter plot highlights vertical trajectories within the flammability limits $\left(\mathrm{Z}_{l}=0.0228<\mathrm{Z}<\right.$ $\left.\mathrm{Z}_{r}=0.1043\right)$ that characterize stratified premixed combustion regime as explained in [17]. At $\mathrm{z}=5 \mathrm{D}$, some chemical trajectories leave the flammability limits and partially-premixed regimes are less important until non-premixed combustion regime is reached at $\mathrm{z}=15 \mathrm{D}$. Low temperature samples around the stoichiometry are present at $\mathrm{z}=15 \mathrm{D}$. These points are attributed to extinction events [17], justified by an operating condition close to the blow-off limit (the bulk main jet velocity is set to $70 \%$ of the blow-off velocity).

Comparisons between numerical and experimental scatter plots show that both FPI and virtual chemistry simulations capture well the thermal flame structure in mixture fraction space. Virtual chemistry performs however slightly better in the near-stoichimoetry high temperature region, especially for the non-premixed flame regime observed at $\mathrm{z}=15 \mathrm{D}$. This observation is consistent with the a-priori analysis conducted on laminar flames [27], that demonstrated the limitations of premixed-based flame tabulation to capture diffusion flame regime. Extinction phenomena are qualitatively well repro- 
duced by numerical simulations both with FPI and virtual chemistry models.

\subsection{CO mass fraction}

Numerical profiles of mean and RMS of CO mass fraction are compared against experimental measurements in Fig. 6. Uncertainties on pilot gas composition at the exit of the burner cause an important departure at $\mathrm{z}=1 \mathrm{D}$. Once pilot gases reach equilibrium, the agreement is significantly improved, especially at $\mathrm{z}=5 \mathrm{D}$ downstream, where temperature and mixing are also correctly predicted. As for the temperature, discrepancy between simulations observed at $\mathrm{z}=15 \mathrm{D}$ are attributed to errors in mixing prediction. Virtual chemistry is trained on a learning database made of both premixed and non-premixed flame archetypes unlike FPI database which is composed of premixed flamelets only. Virtual chemistry is therefore expected to perform better than FPI when the flame structure evolves towards diffusion regimes. This is confirmed at $\mathrm{z}=5 \mathrm{D}$ and $\mathrm{z}=15 \mathrm{D}$, where virtual chemistry results are closer to measurements than FPI solutions.

The ability to model chemical trajectories disregarding errors in flow prediction is addressed by analyzing scatter plots of CO mass fraction in the mixture fraction space shown in Fig. 7. Dots are colored by the flame index [36], defined to reach +1 and -1 in premixed and non-premixed combustion regimes, respectively. Values taken by the flame index reveal, as expected, a stratified premixed flame at $\mathrm{z}=1 \mathrm{D}$ but also a diffusion branch in lean and stochiometric environments at 5D and 15D. The flame structure evolves naturally toward a non-premixed combustion regime when moving downstream. Comparison against experimental scatter data shows that in 

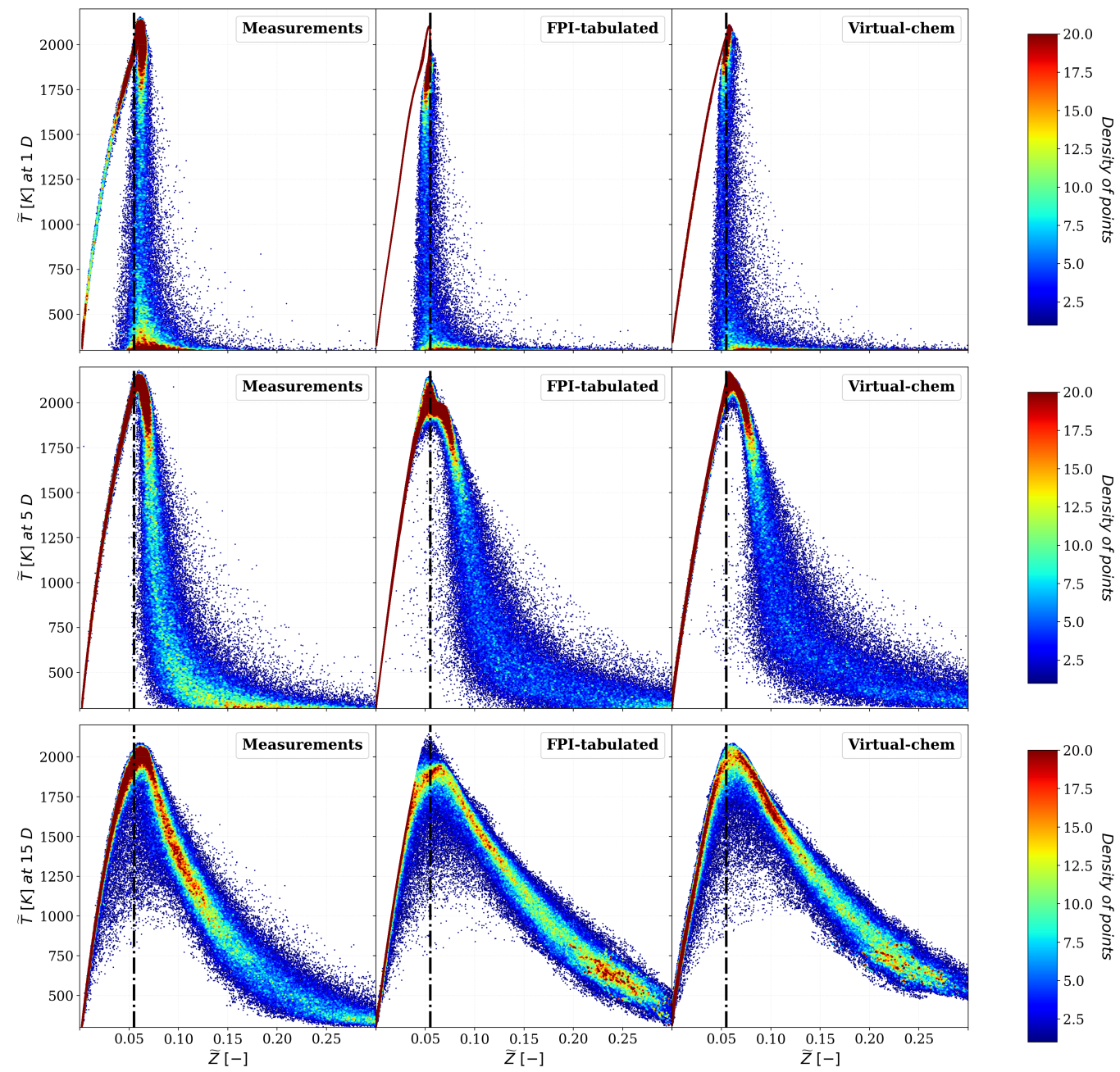

Figure 5: Instantaneous scatter data in temperature-mixture fraction space at three axial planes from the burner exit (From top to bottom: $\mathrm{z} / \mathrm{D}=1,5,15$ ) colored by the local density of points. Experiments are compared to the simulations conducted with FPI and virtual chemistry models. 


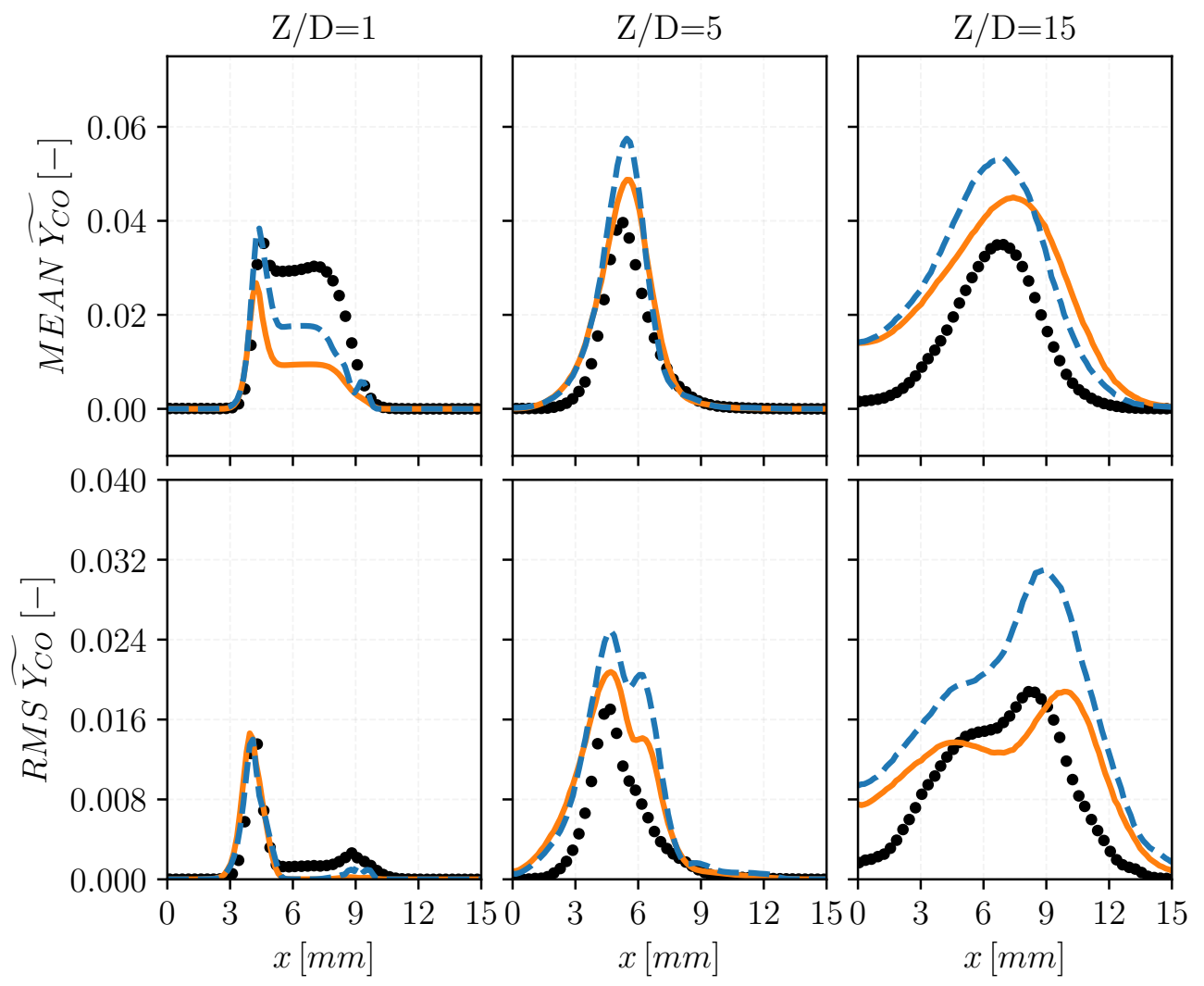

Figure 6: Mean CO mass fraction (top) and RMS (bottom) radial profiles. Comparison of the FPI (Dashed lines) and virtual chemistry (Solid lines) results against experimental data (Circle) at three different distances from the injector. 
diffusion combustion regimes, chemical trajectories are better captured by the virtual chemistry strategy compared to the FPI model. Indeed, unlike to virtual chemistry, FPI does not retrieve the correct distribution in the lean region at $\mathrm{z}=1 \mathrm{D}$ and overpredicts $\mathrm{CO}$ peak at $\mathrm{z}=15 \mathrm{D}$. This overprediction is consistent with the laminar flame computation performed in Sec.3 and plotted in Fig. 1. The $\mathrm{CO}$ is overpredicted by FPI simulations once the flame structure differs from the canonical freely propagating premixed flame. A quantitative comparison between scatter plots is presented in the next section with the introduction of the Wasserstein metric.

\subsection{Wasserstein metric}

The Wasserstein metric $[37,38]$ is a probabilistic measure, useful to quantitatively compare experimental and numerical distributions. It consists in finding the weighted average of the pair-wise distances between samples of two distributions [21]. It has recently been used to evaluate LES turbulent combustion models on the piloted turbulent jet flame with inhomogeneous inlets configuration [21]. Here, the multi-scalar $2^{\text {nd }}$ Wasserstein metric [39] is computed, using the code provided in [21]. Numerical and experimental scatter data constitute two empirical distributions $N$ and $E$, respectively. Each element of the empirical distributions $n_{i}$ and $e_{j}$ contains information for three considered scalars: the mixture fraction $Z$, the temperature $T$ and the CO mass fraction $Y_{C O}$. Data are normalized by the standard deviation of the experimental distribution to give appropriate importance to all retained scalars [21]. The Wasserstein metric is here applied on the piloted turbulent jet flame at two axial locations corresponding to $\mathrm{z} / \mathrm{D}=5,15$. Experimental and numerical scatter data are downsampled by selecting 1000 points, each 


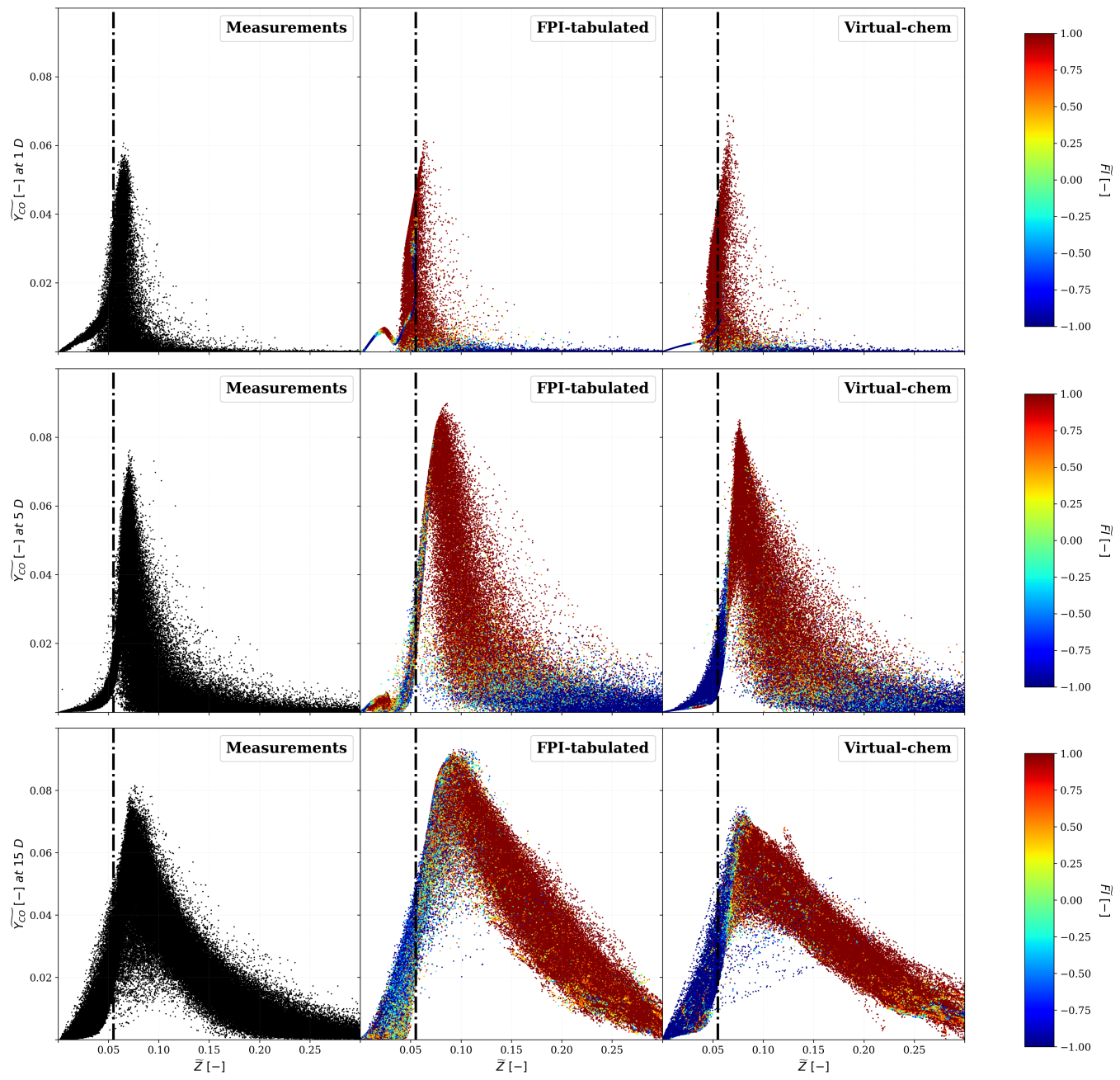

Figure 7: Instantaneous scatter data in $\mathrm{CO}$ mass fraction-mixture fraction space at three axial planes from the burner exit (From top to bottom: $\mathrm{z} / \mathrm{D}=1,5,15$ ). Numerical data are colored by the flame index. Experimental data are compared with the simulations conducted with FPI and virtual chemistry models. 
containing information for $Z, T$ and $Y_{C O}$. The $2^{\text {nd }}$ Wasserstein metric is computed as solution of the following minimization problem:

$$
W_{2}\left(Z, T, Y_{C O}\right)=\min _{\Gamma}\left(\sum_{i=1}^{n} \sum_{j=1}^{n^{\prime}} \gamma_{i j} c_{i j}\right)^{1 / 2}
$$

where $\Gamma$ is the optimal transport matrix having elements $\gamma_{i j}$ and dimension $n \times n^{\prime}$. The unit transportation cost $c_{i j}$ is defined as the $2^{n d}$ power of the pair-wise Euclidean distance: $c_{i j}=\sum_{v=1}^{N_{v}}\left(n_{v, i}-e_{v, j}\right)^{2}$, where $\mathrm{v}$, is the $\mathrm{v}^{\text {th }}$ of variable investigated, and $N_{v}=3$ is the total number of scalar quantities.

Figure 8 shows the cumulative Wasserstein metric for $\mathrm{z} / \mathrm{D}=5,15$ for both FPI and virtual chemistry models. The metric is decomposed in the single variable contributions (different colors) to better analyse the results. The cumulative $W_{2}$ decreases with the axial evolution with both combustion models. This trend is due to the decay of the turbulence and mixing intensity as explained by [21]. The cumulative performance of the virtual chemistry model is better than tabulated chemistry one, in accordance with the scatter data qualitative results. Furthermore, the variable contribution separation helps to conclude that the better virtual chemistry performance is mainly due to an improve prediction of $\mathrm{Y}_{C O}$.

\section{Conclusion}

This article challenged the ability of two reduced chemistry models to capture multi-regime combustion in laminar and turbulent configurations. Whereas only premixed flamelet ingredients in the range of flammability limits are considered in FPI chemical look-up tables, virtual chemistry is optimized to recover the chemical structure of both premixed and non-premixed 


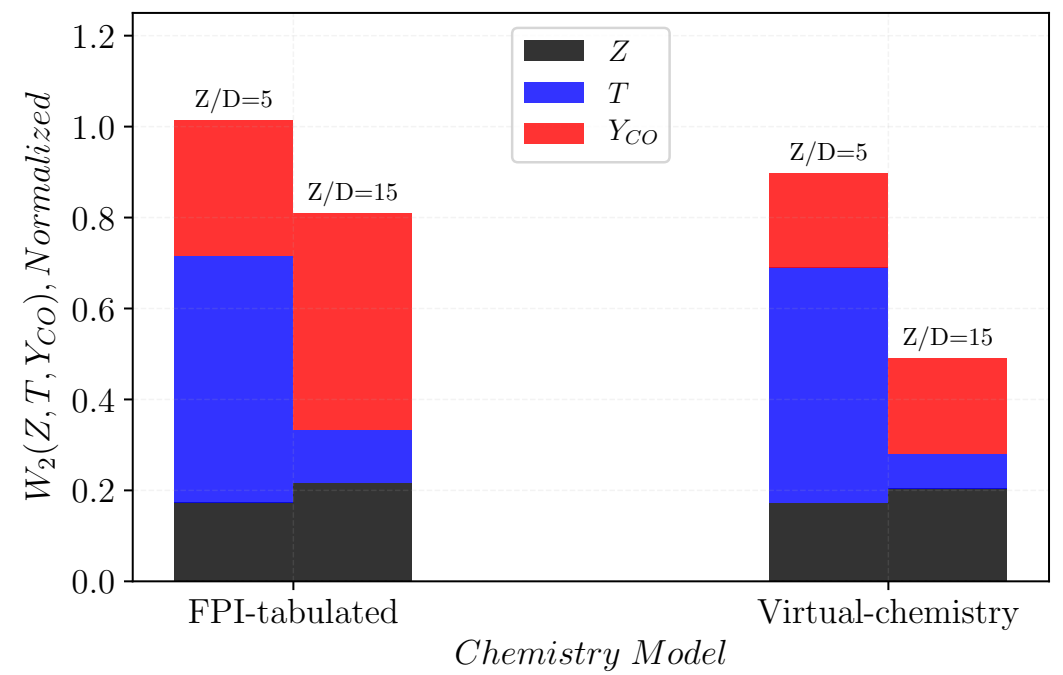

Figure 8: Normalized cumulative multi-scalar $2^{\text {nd }}$ Wasserstein metric for two different axial locations z $/ \mathrm{D}=5,15 . W_{2}\left(Z, T, Y_{C O}\right)$ is compared for the two combustion chemistry models.

flame elements. This is not discriminating in predicting temperature in laminar or turbulent multi-regime flame configurations. Differences are however much more pronounced in the prediction of pollutant formation. In particular, analyses of both laminar and turbulent flame solutions show that virtual chemistry performs better than FPI model on CO mass fraction prediction. The scatter data post-processing, together with the Wasserstein metric, quantitatively confirm the better performance of the virtual chemistry model mainly for $\mathrm{CO}$ prediction in turbulent configuration.

\section{Acknowledgments}

The authors acknowledge Prof. A.R. Masri and Dr. R. Barlow for providing experimental data and B. A. Perry and M.E. Mueller for provid- 
ing the boundary conditions. This project has received funding from the European Union's under the Marie Sklodowska-Curie grant agreement No 643134. This work was granted access to the HPC resources under the allocations A0032B10253 made available by GENCI (Grand Equipement National de Calcul Intensif). This work was performed using HPC resources from the "Mésocentre" computing center of CentraleSupélec and École Normale Supérieure Paris-Saclay supported by CNRS and Région Île-de-France (http://mesocentre.centralesupelec.fr/)

\section{References}

[1] A. Masri, Partial premixing and stratification in turbulent flames, Proc. Combust. Inst. 35 (2015) 1115-1136.

[2] B. Fiorina, D. Veynante, S. Candel, Modeling combustion chemistry in large eddy simulation of turbulent flames, Flow, Turb. and Combustion $94(2015) 3-42$.

[3] T. Jaravel, E. Riber, B. Cuenot, G. Bulat, Large eddy simulation of an industrial gas turbine combustor using reduced chemistry with accurate pollutant prediction, Proceedings of the Combustion Institute 36 (2017) $3817-3825$.

[4] A. Felden, L. Esclapez, E. Riber, B. Cuenot, H. Wang, Including real fuel chemistry in les of turbulent spray combustion, Combustion and Flame 193 (2018) 397 - 416.

[5] N. Peters, Laminar flamelet concepts in turbulent combustion, Symposium (International) on Combustion 21 (1988) 1231-1250. 
[6] U. Maas, S. B. Pope, Simplifying chemical kinetics: intrinsic lowdimensional manifolds in composition space, Combust. Flame 88 (1992) 239-264.

[7] O. Gicquel, N. Darabiha, D. Thévenin, Liminar premixed hydrogen/air counterflow flame simulations using flame prolongation of ILDM with differential diffusion, Proc. Combust. Inst. 28 (2000) 1901-1908.

[8] J. Van Oijen, F. Lammers, L. De Goey, Modeling of complex premixed burner systems by using flamelet-generated manifolds, Combust. Flame 127 (2001) 2124-2134.

[9] V. Bykov, U. Maas, The extension of the ILDM concept to reactiondiffusion manifolds, Combust. Theory and Modelling 11 (2007) 839862.

[10] P.-D. Nguyen, L. Vervisch, V. Subramanian, P. Domingo, Multidimensional flamelet-generated manifolds for partially premixed combustion, Combust. Flame 157 (2010) 43-61.

[11] B. Franzelli, B. Fiorina, N. Darabiha, A tabulated chemistry method for spray combustion, Proc. Combust. Inst. 34 (2013) 1659-1666.

[12] M. Cailler, N. Darabiha, D. Veynante, B. Fiorina, Building-up virtual optimized mechanism for flame modeling, Proc. Combust. Inst. 36 (2017) 1251-1258.

[13] M. Cailler, N. Darabiha, B. Fiorina, Development of a virtual optimized chemistry method. application to hydrocarbon/air combustion, Combust. Flame 211 (2020) 281-302. 
[14] G. Maio, M. Cailler, R. Mercier, B. Fiorina, Virtual chemistry for temperature and CO prediction in LES of non-adiabatic turbulent flames, Proc. Combust. Inst. 37 (2019) 2591-2599.

[15] S. Meares, A. R. Masri, A modified piloted burner for stabilizing turbulent flames of inhomogeneous mixtures, Combust. Flame 161 (2014) 484-495.

[16] S. Meares, V. Prasad, G. Magnotti, R. Barlow, A. Masri, Stabilization of piloted turbulent flames with inhomogeneous inlets, Proc. Combust. Inst. 35 (2015) 1477-1484.

[17] R. Barlow, S. Meares, G. Magnotti, H. Cutcher, A. Masri, Local extinction and near-field structure in piloted turbulent ch 4/air jet flames with inhomogeneous inlets, Combust. Flame 162 (2015) 3516-3540.

[18] https://tnfworkshop.org/workshop proceedings/ (2018).

[19] H. Wu, M. Ihme, Compliance of combustion models for turbulent reacting flow simulations, Fuel 186 (2016) 853-863.

[20] K. Kleinheinz, T. Kubis, P. Trisjono, M. Bode, H. Pitsch, Computational study of flame characteristics of a turbulent piloted jet burner with inhomogeneous inlets, Proc. Combust. Inst. 36 (2017) 1747-1757.

[21] R. Johnson, H. Wu, M. Ihme, A general probabilistic approach for the quantitative assessment of les combustion models, Combust. Flame 183 (2017) 88-101. 
[22] B. A. Perry, M. E. Mueller, A. R. Masri, A two mixture fraction flamelet model for large eddy simulation of turbulent flames with inhomogeneous inlets, Proc. Combust. Inst. 36 (2017) 1767-1775.

[23] S. Galindo, F. Salehi, M. Cleary, A. Masri, Mmc-les simulations of turbulent piloted flames with varying levels of inlet inhomogeneity, Proc. Combust. Inst. 36 (2017) 1759-1766.

[24] B. A. Perry, M. E. Mueller, Effect of multiscalar subfilter pdf models in les of turbulent flames with inhomogeneous inlets, Proc. Combust. Inst. (2018).

[25] L. Tian, R. Lindstedt, Evaluation of reaction progress variable-mixture fraction statistics in partially premixed flames, Proc. Combust. Inst. 37 (2019) 2241-2248.

[26] M. Rieth, J.-Y. Chen, S. Menon, A. M. Kempf, A hybrid flamelet finiterate chemistry approach for efficient les with a transported fdf, Combust. Flame 199 (2019) 183-193.

[27] B. Fiorina, O. Gicquel, L. Vervisch, S. Carpentier, N. Darabiha, Approximating the chemical structure of partially premixed and diffusion counterflow flames using FPi flamelet tabulation, Combust. Flame 140 (2005) 147-160.

[28] G. P. Smith, D. M. Golden, M. Frenklach, B. Eiteener, M. Goldenberg, C. T. Bowman, R. K. Hanson, W. C. Gardiner, V. V. Lissianski, Z. W. Qin, http://www.me.berkeley.edu/gri_mech (2011). 
[29] N. Darabiha, Transient behaviour of laminar counterflow hydrogen-air diffusion flames with complex chemistry, Combust. Sci. Techol. 86 (1992) 163-181.

[30] V. Moureau, P. Domingo, L. Vervisch, Design of a massively parallel cfd code for complex geometries, Comptes Rendus Mécanique 339 (2011) $141-148$.

[31] F. Nicoud, F. Ducros, Subgrid-scale stress modelling based on the square of the velocity gradient tensor, Flow, Turb. and Combustion 62 (1999) 183-200.

[32] O. Colin, F. Ducros, D. Veynante, T. Poinsot, A thickened flame model for large eddy simulations of turbulent premixed combustion, Phys. Fluids 12 (2000) 1843-1863.

[33] J.-P. Legier, T. Poinsot, D. Veynante, in: Proc. of the summer program, Center for Turbulence Research, NASA Ames/Stanford Univ., pp. 157168.

[34] F. Charlette, C. Meneveau, D. Veynante, A power-law flame wrinkling model for les of premixed turbulent combustion part i: non-dynamic formulation and initial tests, Combust. Flame 131 (2002) 159-180.

[35] T. Guiberti, M. Juddoo, D. Lacoste, M. Dunn, W. L. Roberts, A. Masri, Fuel effects on the stability of turbulent flames with compositionally inhomogeneous inlets, Proceedings of the Combustion Institute 36 (2017) $1777-1784$. 
[36] H. Yamashita, M. Shimada, T. Takeno, A numerical study on flame stability at the transition point of jet diffusion flames, Symposium (International) on Combustion 26 (1996) 27 - 34 .

[37] R. M. Dudley, Probabilities and metrics: Convergence of laws on metric spaces, with a view to statistical testing, volume 45, Aarhus Universitet, Matematisk Institut, 1976.

[38] O. Pele, M. Werman, in: 2009 IEEE 12th International Conference on Computer Vision, IEEE, pp. 460-467.

[39] Z. Su, Y. Wang, R. Shi, W. Zeng, J. Sun, F. Luo, X. Gu, Optimal mass transport for shape matching and comparison, IEEE transactions on pattern analysis and machine intelligence 37 (2015) 2246-2259. 